PROCEEDINGS OF THE

AMERICAN MATHEMATICAL SOCIETY

Volume 130, Number 11, Pages 3159-3164

S 0002-9939(02)06436-5

Article electronically published on March 14, 2002

\title{
FINITE HOMOLOGICAL DIMENSION AND PRIMES ASSOCIATED TO INTEGRALLY CLOSED IDEALS
}

\author{
SHIRO GOTO AND FUTOSHI HAYASAKA
}

(Communicated by Wolmer V. Vasconcelos)

\begin{abstract}
Let $I$ be an integrally closed ideal in a commutative Noetherian ring $A$. Then the local ring $A_{\mathfrak{p}}$ is regular (resp. Gorenstein) for every $\mathfrak{p} \in \operatorname{Ass}_{A} A / I$ if the projective dimension of $I$ is finite (resp. the Gorenstein dimension of $I$ is finite and $A$ satisfies Serre's condition $\left(\mathrm{S}_{1}\right)$ ).
\end{abstract}

\section{INTRODUCTION}

Let $I$ be an ideal in a commutative Noetherian ring $A$ and let $x \in A$. Then we say that $x$ is integral over $I$ if there exists an equation $x^{n}+c_{1} x^{n-1}+\cdots+c_{n}=0$ in $A$ with $c_{i} \in I^{i}$. The elements of $A$ which are integral over $I$ form an ideal in $A$ that we denote by $\bar{I}$. We naturally have $I \subseteq \bar{I}$ and the ideal $I$ is said to be integrally closed if $I=\bar{I}$. The purpose of this paper is to study the structure of primes associated to integrally closed ideals possessing finite homological dimension. For a finitely generated $A$-module $M$ let $\operatorname{pd}_{A} M$ (resp. G- $\operatorname{dim}_{A} M$ ) denote the projective (resp. Gorenstein) dimension of $M$. (See Section 3 for the definition of Gorenstein dimension.) Then we have the following.

Theorem (1.1). Let I be an integrally closed ideal in A. Then:

(1) (L. Burch) $A_{\mathfrak{p}}$ is a regular local ring for every $\mathfrak{p} \in \operatorname{Ass}_{A} A / I$ if $\operatorname{pd}_{A} I<\infty$.

(2) Suppose that A satisfies Serre's condition $\left(\mathrm{S}_{1}\right)$ or I contains a non-zerodivisor of $A$. Then $A_{\mathfrak{p}}$ is a Gorenstein local ring for every $\mathfrak{p} \in \operatorname{Ass}_{A} A / I$ if $\mathrm{G}-\operatorname{dim}_{A} I<\infty$.

Theorem (1.1) (1) was first proven by L. Burch [B, p. 947, Corollary 3]; she used the term analytically closed ideal, but it is exactly the same as the integrally closed one. Our method of proof is totally different from hers and it is applicable to explore the primes associated to integrally closed ideals of finite homological dimension of many different kinds, which we shall discuss also in the forthcoming papers [GH1], GHI].

Our research started from a question raised by K. Yoshida of Nagoya University, who asked whether a Noetherian local ring $(A, \mathfrak{m})$ is regular if $A$ contains an

Received by the editors January 1, 2001 and, in revised form, June 8, 2001.

2000 Mathematics Subject Classification. Primary 13H05; Secondary 13H10.

Key words and phrases. Projective dimension, Gorenstein dimension, integrally closed ideal, $\mathfrak{m}$-full ideal, regular local ring, Gorenstein local ring.

The first author was supported by the Grant-in-Aid for Scientific Researches in Japan $(\mathrm{C}(2)$, No. 13640044). 
$\mathfrak{m}$-primary integrally closed ideal possessing finite projective dimension. In $\mathrm{YW}$ Remark (1.13)] he gave an alternative proof in the case where the local rings $A$ are two-dimensional and of positive characteristic. As for the analysis of the corresponding question in the positive characteristic case, the reader may also consult with I. M. Aberbach A Theorem 1.1 and Proposition 2.2], who proved that a Noetherian local ring $(A, \mathfrak{m})$ must be Cohen-Macaulay and F-rational, if $A$ contains a tightly closed ideal $I$ such that $\operatorname{pd}_{A} I<\infty$ and $\mathfrak{m} \in \operatorname{Ass}_{A} A / I$, provided $A$ is equidimensional and a homomorphic image of a Cohen-Macaulay ring of positive characteristic.

We shall give a proof of Theorem (1.1) in Section 3. For that purpose, passing to the ring $A_{\mathfrak{p}}$, we may assume that $(A, \mathfrak{m})$ is a local ring and $\mathfrak{m} \in \operatorname{Ass}_{A} A / I$. Our aim is the regularity and the Gorensteinness of $A$. Hence, passing to the ring $A[X]_{\mathfrak{m} A[X]}$ with $X$ an indeterminate over $A$, we may also assume without loss of generality that the field $A / \mathfrak{m}$ is infinite. Then since $I=\bar{I}$, by [G2, Theorem (2.4)] we have $I=\sqrt{(0)}$ or $\mathfrak{m} I: x=I$ for some $x \in \mathfrak{m}$. The ideals $I$ satisfying the latter condition are called $\mathfrak{m}$-full. (Our definition of $\mathfrak{m}$-full ideals is slightly different from the one which was given by [W1], W2].) The notion of $\mathfrak{m}$-full ideal was introduced by D. Rees and some results on $\mathfrak{m}$-full ideals will play a crucial role in our argument, which we shall briefly summarize in Section 2 (Proposition (2.2) and Corollary $(2.3))$.

\section{PReliminaries ON m-FUll IDEALS}

Let $A$ be a Noetherian local ring with the maximal ideal $\mathfrak{m}$. The purpose of this section is to give some auxiliary results on $\mathfrak{m}$-full ideals that we need to prove Theorem (1.1). Let $\mu_{A}(*)$ stand for the number of generators and $\ell_{A}(*)$ the length. We begin with the following.

Lemma (2.1). Let $I$ be an $\mathfrak{m}$-primary ideal in $A$ and let $x \in \mathfrak{m}$. Then $\mathfrak{m} I: x=I$ if and only if $\mu_{A}(I)=\ell_{A}(A /(I+(x)))+\mu_{A}((I+(x)) /(x))$.

Proof. See [G2, Lemma (2.2) and the proof].

The next is the key for our proof of Theorem (1.1).

Proposition (2.2). Let $I$ be an ideal in $A$ and assume that $\mathfrak{m} I: x=I$ for some $x \in \mathfrak{m}$. Let $\ell=\ell_{A}((I: \mathfrak{m}) / I)$ and write $I: \mathfrak{m}=\left(y_{1}, y_{2}, \ldots, y_{\ell}\right)+I$ with $y_{i} \in I: \mathfrak{m}$. Then the following assertions hold true:

(1) $I: \mathfrak{m}=I: x$.

(2) $x \notin \mathfrak{m}^{2}$ if $\mathfrak{m} \in \operatorname{Ass}_{A} A / I$.

(3) The elements $\left\{x y_{i}\right\}_{1 \leq i \leq \ell}$ constitute a part of a minimal basis of $I$.

(4) Let $I=\left(x y_{1}, x y_{2}, \ldots, x y_{\ell}\right)+\left(z_{1}, z_{2}, \ldots, z_{m}\right)$ with $z_{j} \in I$ and $\ell+m=\mu_{A}(I)$. Then

$$
I / x I=\sum_{i=1}^{\ell} A \cdot \overline{x y_{i}} \oplus \sum_{j=1}^{\mathfrak{m}} A \cdot \overline{z_{j}}
$$

and $\ell_{A}\left(\sum_{i=1}^{\ell} A \cdot \overline{x y_{i}}\right)=\ell$, where $\bar{*}$ denotes the reduction $\bmod x I$.

Proof. See [W1, Lemma 1] for assertion (1). Suppose that $x \in \mathfrak{m}^{2}$. Then $I=$ $\mathfrak{m} I: x \supseteq \mathfrak{m} I: \mathfrak{m}^{2}=(\mathfrak{m} I: \mathfrak{m}): \mathfrak{m}$, so that $I \supseteq I: \mathfrak{m}$ whence $\mathfrak{m} \notin \operatorname{Ass}_{A} A / I$. Let 
$\left\{a_{i}\right\}_{1 \leq i \leq \ell}$ be elements in $A$ such that $\sum_{i=1}^{\ell} a_{i}\left(x y_{i}\right) \in \mathfrak{m} I$. Then $\sum_{i=1}^{\ell} a_{i} y_{i} \in \mathfrak{m} I$ : $x=\bar{I}$ and so we have $a_{i} \in \mathfrak{m}$ for all $1 \leq i \leq \ell$. Thus $\left\{x y_{i}\right\}_{1 \leq i \leq \ell}$ is a part of a minimal basis of $I$. Let $\left\{a_{i}\right\}_{1 \leq i \leq \ell}$ and $\left\{b_{j}\right\}_{1 \leq j \leq m}$ be elements in $A$ and assume that $\sum_{i=1}^{\ell} a_{i}\left(x y_{i}\right)+\sum_{j=1}^{m} b_{j} z_{j} \in x I$. Then since $\left\{x y_{i}\right\}_{1 \leq i \leq \ell}$ and $\left\{z_{j}\right\}_{1 \leq j \leq m}$ form a minimal basis of $I$, we get $a_{i}, b_{j} \in \mathfrak{m}$ for any $i$ and $j$, so that $a_{i} y_{i} \in I$ for all $1 \leq i \leq \ell$. Consequently $\sum_{i=1}^{\ell} a_{i}\left(x y_{i}\right) \in x I$, whence $\sum_{j=1}^{m} b_{j} z_{j} \in x I$, too. Thus $I / x I=\sum_{i=1}^{\ell} A \cdot \overline{x y_{i}} \oplus \sum_{j=1}^{\mathfrak{m}} A \cdot \overline{z_{j}}$. Since $\mathfrak{m} \cdot\left(x y_{i}\right) \subseteq x I$ for every $1 \leq i \leq \ell$, this argument also shows that $\ell_{A}\left(\sum_{i=1}^{\ell} A \cdot \overline{x y_{i}}\right)=\ell$ as is asserted.

Let $\beta_{i}^{A}(*)$ stand for Betti numbers. The next result is an easy consequence of Proposition (2.2) and entirely covers [W2, Theorem 6 and Corollary 7]. Let us give a brief proof for completeness.

Corollary (2.3). Let $I$ be an ideal in $A$ and assume that $\mathfrak{m} I: x=I$ for some $x \in \mathfrak{m}$. Then

$$
I / x I \cong(I: \mathfrak{m}) / I \oplus(I+(x)) /(x) \cong(I: \mathfrak{m}) / I \oplus I / x(I: \mathfrak{m}) .
$$

Hence the equality

$$
\beta_{i}^{A}(I)=\ell \cdot \beta_{i-1}^{A /(x)}(\mathfrak{m} /(x))+\beta_{i}^{A /(x)}((I+(x)) /(x))
$$

holds true for all $i \geq 1$ if $x$ is $A$-regular, where $\ell=\ell_{A}((I: \mathfrak{m}) / I)$.

Proof. First we look at the commutative diagram

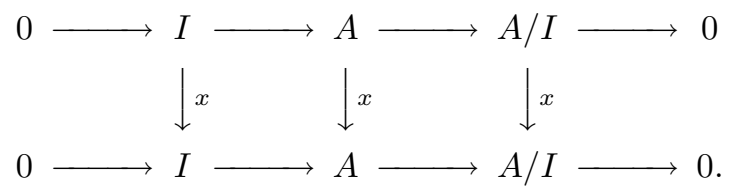

Then since (0) $: x \subseteq \mathfrak{m} I: x=I$ and $I: x=I: \mathfrak{m}$ by (2.2) (1), thanks to the snake lemma we get the exact sequence

$$
0 \rightarrow(I: \mathfrak{m}) / I \stackrel{f}{\rightarrow} I / x I \rightarrow A /(x) \rightarrow A /(I+(x)) \rightarrow 0
$$

of $A$-modules. Let $I: \mathfrak{m}=\left(y_{1}, y_{2}, \ldots, y_{\ell}\right)+I$ with $y_{i} \in I: \mathfrak{m}$ for all $1 \leq i \leq \ell$. Then since $f(y \bmod I)=x y \bmod x I$ for each $y \in I: \mathfrak{m}$, we have that $\operatorname{Im} f=\sum_{i=1}^{\ell} A \cdot \bar{x} y_{i}$ in $I / x I$, whence by $(2.2)$ (4) the short exact sequence $0 \rightarrow(I: \mathfrak{m}) / I \stackrel{f}{\rightarrow} I / x I \rightarrow$ $(I+(x)) /(x) \rightarrow 0$ splits and the decompositions

$$
I / x I \cong(I: \mathfrak{m}) / I \oplus(I+(x)) /(x) \cong(I: \mathfrak{m}) / I \oplus I / x(I: \mathfrak{m})
$$

of $I / x I$ readily follow. (Recall that $(I+(x)) /(x) \cong I / x(I: x)=I / x(I: \mathfrak{m})$ by (2.2) (1).) The second assertion is clear, since $\beta_{i}^{A /(x)}(I / x I)=\beta_{i}^{A}(I)$ for all $i \geq 0$ if $x$ is $A$-regular.

Let us add one more result, which is closely related to [CHV Theorem 3.7]. In the forthcoming paper [GH2] we shall study the structure of the set $\mathcal{S}_{A}$ of integrally closed m-primary ideals $I$ of $A$ such that $A / I$ are Gorenstein. The next result will play a key role in it. 
Proposition (2.4). Let $I$ be an $\mathfrak{m}$-primary ideal in $A$ and let $n=\mu_{A}(I)$. Then the following two conditions are equivalent:

(1) $I$ is $\mathfrak{m}$-full, that is $\mathfrak{m} I: x=I$ for some $x \in \mathfrak{m}$, and $A / I$ is a Gorenstein ring.

(2) $\mu_{A}(\mathfrak{m})=n \geq 1$ and there exists a minimal basis $\left\{a_{i}\right\}_{1 \leq i \leq n}$ of $\mathfrak{m}$ such that $I=\left(a_{1}, \ldots, a_{n-1}, a_{n}^{q}\right)$ for some $q \geq 1$.

When this is the case, the integer $q$ in assertion (2) is given by

$$
q=\ell_{A}(A / I)=\min \left\{1 \leq s \in \mathbb{Z} \mid \mathfrak{m}^{s} \subseteq I\right\},
$$

which is independent of the choice of the minimal basis $\left\{a_{i}\right\}_{1 \leq i \leq n}$ for $\mathfrak{m}$.

Proof. (1) $\Rightarrow(2)$ Let $x \in \mathfrak{m}$ such that $\mathfrak{m} I: x=I$. Then $x \notin \mathfrak{m}^{2}$ by (2.2) (2). Let $I: \mathfrak{m}=I+(y)$ with $y \in I: \mathfrak{m}$. Then $x y \notin \mathfrak{m} I$ as $y \notin I$, whence $n \geq 1$. We write $I=\left(a_{1}, \ldots, a_{n-1}, x y\right)$ with $a_{i} \in I$. Then $\mu_{A}((I+(x)) /(x))=n-1$ by $(2.3)$ and $n=\ell_{A}(A /(I+(x)))+\mu_{A}((I+(x)) /(x))$ by $(2.1)$. Hence $\ell_{A}(A /(I+(x)))=1$ so that we have $\mathfrak{m}=I+(x)=\left(a_{1}, \ldots, a_{n-1}, x\right)$. Therefore $\mu_{A}(\mathfrak{m})=n$, because $x \notin \mathfrak{m}^{2}$ and the maximal ideal $\mathfrak{m} /(x)=(I+(x)) /(x)$ in the local ring $A /(x)$ is minimally generated by $n-1$ elements. Let $J=\left(a_{1}, a_{2}, \ldots, a_{n-1}\right)$ and let $*$ denote the reduction $\bmod J$. Then $\mathfrak{m} / J=(\bar{x})$ and $x y \notin J$, because $J \subsetneq I$ (recall that $\mu_{A}(I)=n$ ). Thus every non-zero ideal in the local ring $A / J$ is a power of the maximal ideal $\mathfrak{m} / J$, whence $(\overline{x y})=\left(\bar{x}^{q}\right)$ for some $q \geq 1$. Thus $I=J+(x y)=J+\left(x^{q}\right)$ as is claimed.

$(2) \Rightarrow(1)$ Let $x=a_{n}$. We have

$$
\ell_{A}(A /(I+(x)))+\mu_{A}((I+(x)) /(x))=\ell_{A}(A / \mathfrak{m})+\mu_{A}(\mathfrak{m} /(x))=1+(n-1)=n,
$$

so that $\mathfrak{m} I: x=I$ by (2.1). Hence $I$ is $\mathfrak{m}$-full. The Artinian local ring $A / I$ is a principal ideal ring because its maximal ideal $\mathfrak{m} / I$ is cyclic, whence it is a Gorenstein ring.

To see the last assertion, let $s=\min \left\{1 \leq s \in \mathbb{Z} \mid \mathfrak{m}^{s} \subseteq I\right\}$ and $\ell=\ell_{A}(A / I)$. Then $\mathfrak{m}^{q} \subseteq I$ because $a_{n}^{q} \in I$, whence $s \leq q$. We also have that $\ell=s$, since $A / I$ is a principal ideal ring and every ideal in it is a power of the maximal ideal $\mathfrak{m} / I$. Assume that $s<q$ and write $a_{n}^{s}=j+c a_{n}^{q}$ with $j \in J=\left(a_{1}, a_{2}, \ldots, a_{n-1}\right)$ and $c \in A$. Then because $a_{n}^{s}\left(1-c a_{n}^{q-s}\right) \in J$, we get $a_{n}^{s} \in J$ so that $a_{n}^{q} \in J$, which is impossible since $I \neq J$. Thus the equalities $q=\ell=s$ follow.

\section{Proof of Theorem (1.1)}

The notion of Gorenstein dimension is a generalization of projective dimension and was introduced by M. Auslander [Au]. Since it might be less familiar to readers, before entering the proof of Theorem (1.1), let us recall the definition of Gorenstein dimension and some properties as well.

Let $A$ be a commutative Noetherian ring and let $\mathcal{C}$ denote the class of finitely generated reflexive $A$-modules $X$ such that $\operatorname{Ext}_{A}^{i}(X, A)=\operatorname{Ext}_{A}^{i}\left(X^{*}, A\right)=(0)$ for all $i \geq 1$, where $X^{*}=\operatorname{Hom}_{A}(X, A)$. Then the class $\mathcal{C}$ contains all the finitely generated projective $A$-modules and for a given finitely generated $A$-module $M$ the Gorenstein dimension $\mathrm{G}-\operatorname{dim}_{A} M$ of $M$ is defined to be the minimum of integers $n \geq 0$ for which there exists an exact sequence

$$
0 \rightarrow X_{n} \rightarrow X_{n-1} \rightarrow \cdots \rightarrow X_{0} \rightarrow M \rightarrow 0
$$

of length $n$ with $X_{i} \in \mathcal{C}$. Therefore we naturally have that $\mathrm{G}_{-} \operatorname{dim}_{B} B \otimes_{A} M \leq$ G-dim ${ }_{A} M$ for every flat Noetherian $A$-algebra $B$ and that $\operatorname{Ext}_{A}^{i}(M, A)=(0)$ for all $i \gg 0$ if $\mathrm{G}-\operatorname{dim}_{A} M<\infty$. 
The basic properties of Gorenstein dimension are as follows.

Proposition (3.1) ([A] $)$. Let $A$ be a commutative Noetherian ring. Then the following assertions hold true:

(1) Let $0 \rightarrow M_{1} \rightarrow M_{2} \rightarrow M_{3} \rightarrow 0$ be a short exact sequence of finitely generated A-modules. If two of the $M_{i}$ 's have finite Gorenstein dimension, then so does the other.

(2) Let $M$ be a finitely generated A-module. Then

$$
\text { G- } \operatorname{dim}_{A} N \leq \mathrm{G}-\operatorname{dim}_{A} M
$$

for every direct summand $N$ of $M$.

(3) Assume that $A$ is a local ring and let $M$ be a finitely generated non-zero A-module. Then

$$
\operatorname{depth} A=\operatorname{depth}_{A} M+\mathrm{G}-\operatorname{dim}_{A} M
$$

if $\mathrm{G}-\operatorname{dim}_{A} M<\infty$. Hence $\mathrm{G}-\operatorname{dim}_{A} M=\operatorname{pd}_{A} M$ if $\operatorname{pd}_{A} M<\infty$.

(4) Assume that $(A, \mathfrak{m})$ is a local ring. Then $A$ is a Gorenstein ring if and only if $\mathrm{G}-\operatorname{dim}_{A} A / \mathfrak{m}<\infty$. When this is the case, we have $\mathrm{G}-\operatorname{dim}_{A} M<\infty$ for every finitely generated $A$-module $M$.

Proposition (3.2) ([G1]). Let $A$ be a (not necessarily local) Gorenstein ring. Then G- $\operatorname{dim}_{A} M<\infty$ for every finitely generated $A$-module $M$.

We are now in a position to prove Theorem (1.1).

Proof of Theorem (1.1). We may assume the base $\operatorname{ring}(A, \mathfrak{m})$ is local and $\mathfrak{m} \in$ $\operatorname{Ass}_{A} A / I$. We want to show that $A$ is regular or Gorenstein according to cases (1) and (2). Passing to the ring $A[X]_{\mathfrak{m} A[X]}$ with $X$ an indeterminate over $A$, we may assume the field $A / \mathfrak{m}$ is infinite. Hence by [G2, Theorem (2.4)] we have $I=\sqrt{(0)}$ or $\mathfrak{m} I: x=I$ for some $x \in \mathfrak{m}$, because $I=\bar{I}$. The proof of [G2, Theorem (2.4)] also guarantees that if $\operatorname{depth} A>0$ and $\mathrm{I} \neq \sqrt{(0)}$, we may choose the element $x \in \mathfrak{m}$ so that $x$ is $A$-regular.

(1) If $I=(0)$, then $A$ is a reduced ring with $\mathfrak{m} \in \operatorname{Ass} A$, whence $A$ is a field. Assume that $I \neq(0)$. Then since $\operatorname{pd}_{A} A / I<\infty$, by $[\mathrm{V}$, Proposition 1.3] the ideal $I$ must contain a regular element of $A$, whence $I \neq \sqrt{(0)}$. Choose $x \in \mathfrak{m}$ so that $x$ is $A$-regular and $\mathfrak{m} I: x=I$. Then $\operatorname{pd}_{A} I / x I<\infty$ because $\operatorname{pd}_{A} I<\infty$, whence by $(2.3) \operatorname{pd}_{A}((I: \mathfrak{m}) / I)<\infty$, too. We have $(I: \mathfrak{m}) / I \neq(0)$ since $\mathfrak{m} \in \operatorname{Ass}_{A} A / I$, so that $\operatorname{pd}_{A} A / \mathfrak{m}<\infty$ and $A$ is a regular local ring.

(2) First suppose that $I=\sqrt{(0)}$. Then $\operatorname{dim} A=0$ since $\mathfrak{m} \in \operatorname{Ass}_{A} A / I$, so that $I=\mathfrak{m}$ because $I=\bar{I}$. Thus $\mathrm{G}-\operatorname{dim}_{A} A / \mathfrak{m}<\infty$ and so by (3.1) (4) $A$ is a Gorenstein local ring. Assume that $I \neq \sqrt{(0)}$. Then $\operatorname{depth} A>0$. (Otherwise, the $\operatorname{ring} A$ must satisfy Serre's condition $\left(\mathrm{S}_{1}\right)$ so that $\operatorname{dim} A=0$, too, whence $I=\mathfrak{m}=\sqrt{(0)}$ which is absurd.) Choose $x \in \mathfrak{m}$ so that $x$ is $A$-regular and $\mathfrak{m} I: x=I$. Then by (3.1) (1) we see that $\mathrm{G}-\operatorname{dim}_{A} I / x I<\infty$ since $\mathrm{G}-\operatorname{dim}_{A} I<\infty$. Thus $\mathrm{G}-\operatorname{dim}_{A} A / \mathfrak{m}<\infty$ by (2.3) and (3.1) (2), whence by (3.1) (4) $A$ is a Gorenstein local ring.

Remark (3.3). The above proof of Theorem (1.1) (1) actually shows that a Noetherian local ring $(A, \mathfrak{m})$ is regular if it contains an $\mathfrak{m}$-full ideal $I$ such that $\operatorname{pd}_{A} I<\infty$ and $\mathfrak{m} \in \operatorname{Ass}_{A} A / I$. 


\section{REFERENCES}

[A] I. M. Aberbach, Tight closure in F-rational rings, Nagoya Math. J. 135 (1994), 43-54. MR 95g:13020

[Au] M. Auslander, Anneaux de Gorenstein et torsion en algèbre commutative, Séminaire d'algèbre commutative dirigé par Pierre Samuel 1966/67, École Normale Supérieure de Jeunes Fillies, 1967. MR 37:1435

[B] L. Burch, On ideals of finite homological dimension in local rings, Proc. Camb. Phil. Soc. 64 (1968), 941-948. MR 37:5208

[CHV] A. Corso, C. Huneke, and W. V. Vasconcelos, On the integral closure of ideals, Manuscripta Math. 95 (1998), 331-347. MR 99b:13010

[G1] S. Goto, Vanishing of $\operatorname{Ext}_{A}^{i}(M, A)$, J. Math. Kyoto Univ. 22 (1982), 481-484. MR 84c:13019

[G2] S. Goto, Integral closedness of complete-intersection ideals, J. Alg. 108 (1987), 151-160. MR 88d:13015

[GH1] S. Goto and F. Hayasaka, Finite homological dimension and primes associated to integrally closed ideals II, Preprint 2001.

[GH2] S. Goto and F. Hayasaka, Gorenstein integrally closed $\mathfrak{m}$-primary ideals, in preparation.

[GHI] S. Goto, F. Hayasaka, and S.-I. Iai, The a-invariant and Gorensteinness of graded rings associated to filtrations of ideals in regular local rings, Proc. Amer. Math. Soc. (to appear).

[V] W. Vasconcelos, Ideals generated by R-sequences, J. Alg. 6 (1967), 309-316. MR 35:4209

[W1] J. Watanabe, m-full ideals, Nagoya Math. J. 106 (1987), 101-111. MR 88g:13003

[W2] J. Watanabe, The syzygies of $\mathfrak{m}$-full ideals, Math. Proc. Cambridge Phil. Soc. 109 (1991), 7-13. MR 92b:13019

[YW] K. Yoshida and K. Watanabe, Hilbert-Kunz multiplicity, McKay correspondence, and good ideals in two-dimensional rational singularities, Manuscripta Math. 104 (2001), 275-294. CMP 2001:11

Department of Mathematics, School of Science and Technology, Meiji University, 214-8571 JAPAN

E-mail address: goto@math.meiji.ac.jp

Department of Mathematics, School of Science and Technology, Meiji University, 214-8571 JAPAN

E-mail address: ee68048@math.meiji.ac.jp 